\title{
MCP-1, ICAM-1 and VCAM-1 are present in early aneurysmal dilatation in experimental rats
}

\author{
Jun Fan ${ }^{1}$, Xiang Li ${ }^{2}$, Linlin Zhong ${ }^{3}$, Hao-Tong ${ }^{1}$, Jing Di ${ }^{1}$, Fang Liu ${ }^{1}$, Hai-Hua Zhao ${ }^{1}$, \\ Shu-Ling Bai ${ }^{1}$
}

${ }^{1}$ Department of Tissue Engineering, Department of Human Anatomy, College of Basic Medicine, China Medical University, Shenyang, China

${ }^{2}$ Department of Cell Biology, College of Basic Medicine, China Medical University, Shenyang, China

${ }^{3}$ Department of Pharmacology, Southern Illinois University School of Medicine, Springfield IL, USA

\begin{abstract}
Recent studies have suggested that inflammation actively participates in ascending aortic aneurysm formation. The aim of the present study was to evaluate the expression changes of adhesion molecules and MMPs in an experimental model of ascending aortic aneurysm induced by ascending aorta banding in Wistar rats. Twelve rats developed aortic dilation after ascending aorta banding treatment, while nine normal animals underwent surgery without banding were used as controls. Light microscope and scanning electron microscope showed that the wall of the ascending aorta became disorganized as well as infiltration by inflammatory cells in aneurysmal rats. By using immunohistochemical techniques, a significant increase in the immunostaining of MCP-1 was observed in the aneurysmal wall as compared to the normal aortic wall. Under similar experimental conditions, we also found that the immunostaining of ICAM-1 and VCAM-1 was markedly increased in the aneurysmal wall. In addition, gelatin zymographic analysis showed that the expression and acitivities of MMP-2 and MMP-9 were remarkably enhanced in the ascending aorta of ascending aortic aneurysmal rats as compared to normal rats. These results demonstrate that MCP-1, ICAM-1 and VCAM-1 are involved in the pathogenesis of ascending aortic aneurysm and an increase in the immunostaining and activity of MMP-2 and MMP-9 may promote the progression of ascending aortic aneurysm.
\end{abstract}

Key words: ascending aortic aneurysm, inflammation, adhesion molecules, MMP, rat

\section{Introduction}

Thoracic aortic aneurysm is an important cause of mortality due to rupture or dissection, in which ascending aortic aneurysm accounts for $40 \%$ of diagnosed patients $[1,2]$. Ascending aortic aneurysm has been shown to be associated with degeneration of the arterial tunica media, congenital cardiovascular malformations, connective tissue disorders, and aortic dissection [3]. Ascending aortic aneurysm initially was described as a non-inflammatory lesion. However, recent evidence indicates that the infiltration of inflammatory cells contributes to the pathogenesis of ascending aortic aneurysms $[4,5]$.

It has been demonstrated that inflammatory markers were associated with cardiovascular disease, sug-

Correspondence: Shu-Ling Bai, Dept. of Tissue Engineering,

China Medical University, Shenyang 110001, P.R. China; tel.: (+8624) 23256666-5295, fax.: (+8624) 23256666-5295, e-mail: baishuling@hotmail.com gesting that inflammation plays a key role in the initiation and progression of cardiovascular disease $[1,6]$. The release of proinflammatory molecules induces the accumulation of monocytes, smooth muscle cells, and lymphocytes within the arterial wall, resulting in the formation of atherosclerotic plaque, eventually leading to complications, such as total artery occlusion, rupture, calcification, or aneurysm $[7,8]$. Aneurysmal and atherosclerotic arteries are characterized by infiltration of leukocytes, particularly macrophages and T cells, which elicit immune responses and vascular disease [9-13]. Infiltrating inflammatory cells release many inflammatory cytokines and promote the production of matrix metalloproteinases (MMPs) [14]. Recent studies have shown that MMPs are related to aortic aneurysm formation, particularly MMP-2 and MMP-9.

Several molecules play a key role in the inflammatory cascade. Adhesion molecules, vascular cell adhesion molecule-1 (VCAM-1) and intercellular adhesion molecule-1 (ICAM-1), play a significant role in ensur- 
ing the recruitment of inflammatory cells. Their role in inflammation is linked to leukocyte attachment, rolling, and trans-endothelial migration. The ICAM-1, an immunoglobulin-like molecule, was expressed very low in endothelial cells, while its expression was markedly increased with exposure to inflammatory cytokines. VCAM-1 has been shown to be involved in accumulation and adhesion of leukocytes primarily by interacting with its counterligand VLA-4 in monocytes and lymphocytes [15]. Monocyte chemoattractant protein-1 (MCP-1) belongs to the CC chemokine family and is a potent agonist for monocytes, $\mathrm{T}$ lymphocytes, natural killer cells, and basophils [16].

However, few studies have focused on inflammatory disease of the proximal aorta and the mechanism for pathogenesis of ascending aortic aneurysm is still not completely understood. A clear understanding of these events is critical for mechanistic and therapeutic exploitation in ascending aortic aneurysm. Our previous studies have demonstrated that ascending aortic dilation was induced by banding the ascending aorta $[17,18]$.

The aim of this study was to follow morphological changes and determine the immunohistochemical localization of MCP-1, ICAM-1 and VCAM-1 in the early aneurysmal dilatation, which could provide evidence that these proteins contribute to the development of ascending aortic aneurysm.

\section{Materials and methods}

Animal use and surgical procedure. Young female Wistar rats (80-100g, provided by the experimental animal center of China Medical University) were used in the study. All animal experiments were carried out in accordance with the ethical standards of China Medical University. The models $(n=12)$ were established as previously described $[17,18]$. Briefly, animals were anesthetized with pentobarbital sodium (40 mg/kg, intraperitoneally) and connected to a rodent ventilator (Jiang Wan II) set at a respiratory rate of 100 strokes per minute with a tidal volume of $3 \mathrm{ml}$. The animal was then carefully rotated to the left lateral decubitus position, exposing the right chest. A sterile thoracotomy was performed between the second and third interspace, the ascending aorta was dissected free of the pulmonary artery, and a flexible polyethylene tube $(7 \mathrm{~mm}$ diameter) with a $4-0$ silk suture was placed around the aortic root (The average perimeter of the ascending aorta is $7 \mathrm{~mm}$ in live young female Wistar rats). After the chest was closed, animals were given an injection of antibiotics agent (Penicillin 10,000 U i.m.) for 3 days. Sham-operated animals $(n=9)$ underwent surgery without banding. About $30 \%$ of animals that underwent surgery died either just after surgery or during the overall period of aneurysmal dilation. The animals were housed in a temperaturecontrolled room under normal $12 \mathrm{~h}$ light $/ 12 \mathrm{~h}$ dark laboratory conditions with free access to chow and water. Previous studies have shown successful induction of aneurysms in the descending aorta of monkeys after the initial procedure [19]. The aneurysmal degeneration could be seen at 8 weeks after the surgery.

Ultrasonography. Ultrasonography was performed to demonstrate dilatation of the ascending aortas. In this study, 21 anesthetized rats were placed on a rat bed in a shallow left lateral decubitus position. Transthoracic echocardiography was performed using a pediatric broadband 6-15 MHz linear array ultrasound transducer. For an accurate measurement of aortic diameter, we calculated aneurysm diameters using SZH light microscopy at aortic harvest.

Harvesting procedures. After ultrasonography detection, the animals were re-anesthetized and the initial incisions were re-opened. The circulatory system was perfused with normal saline. Then the aortas were harvested. The samples were either frozen in liquid nitrogen or fixed in neutral buffered formalin or glutaraldehyde. For each subject, one part of aneurysm was used for histologic examination and the other part of aneurysm was used for gelatin zymography studies.

Staining. Fixing samples were dehydrated through ethanol and embedded in paraffin for sectioning. Aortic tissue cross-sections $(5 \mu \mathrm{m})$ of rats were stained with hematoxylin and eosin (H\&E) for general tissue morphology in the standard manner. The change of elastic lamellar was observed by Romeis staining.

Scanning Electron Microscopy. The samples were postfixed in $2.5 \%$ glutaraldehyde solution for 12 hours, followed by overnight in $0.1 \mathrm{~mol} / \mathrm{L} \mathrm{PBS}(\mathrm{pH} 7.2)$, all at room temperature. The tissues were next dehydrated in ethanol, critical point-dried with $\mathrm{CO}_{2}$ and sputter-coated with gold palladium. A standardized area of each specimen was then examined in a blinded fashion with scanning electron microscope (JSM-T300).

Immunohistochemistry. Anti-CD45 (1:200, Boisynthesis, China), anti-VCAM-1 (1:100, Boster, China), anti-ICAM-1(1:100, Boster, China), anti-MCP-1 (1:100, Santa Cruz Biotech, Santa Cruz, CA, USA) were used for analysis. Immunohistochemical staining was performed using the strept-avidin biotin complex (SABC) test method. Diluted primary antibodies (1:200) were then applied to the sections, and these sections were incubated overnight at $4^{\circ} \mathrm{C}$. Immune complexes were visualized using 3, 3'-diaminobenzidine (DAB), and slides were counterstained with hematoxylin. All sections were then dehydrated in graded alcohols and covered by coverslips with neutral balsam for light microscopy. For negative control experiments, the primary antibody was omitted and processed as described above. Non-specific staining was not observed. For all sections, we assessed the mean optical density.

Gelatin zymography. Frozen aortic tissues were homogenized and total proteins extracted. Tissue proteins were subjected to SDS-PAGE using $7.5 \%$ acrylamide gels containing $0.1 \%$ gelatin. The gels were then incubated for $30 \mathrm{~min}$ at room temperature in $2.5 \%$ Triton $\mathrm{X}-100$, agitated on a rocking bed, and then transferred to activation buffer $\left(50 \mathrm{mM}\right.$ Tris-Cl $\mathrm{pH} 7.5,10 \mathrm{mM} \mathrm{CaCl}_{2}, 200$ $\mathrm{mmol} / \mathrm{L} \mathrm{NaCl}, 1 \mu \mathrm{mol} / \mathrm{L} \mathrm{ZnCl}_{2}$ ) for 18 hours incubation at $37^{\circ} \mathrm{C}$. Gels were then stained with $0.1 \%$ Coomassie Brilliant Blue R-250 for 4 hour and briefly destained in $10 \%$ acetic acid and $40 \%$ methanol for $0.5-1$ hour. Gelatinolytic activity was detected as transparent bands on a blue background. Gels were scanned and integrated density values (IDVs) were calculated by Fluor Chen 2.0 software.

Statistical analysis. SPSS 13.0 statistical software was used for statistical analysis. The Mann Whitney test was used for data analysis, $\mathrm{p}<0.05$ was considered statistically significant.

\section{Results}

\section{Morphologic changes of the ascending aorta in Wistar rats with ascending aortic dilatation}

To investigate the formation mechanism of ascending aortic aneurysm, we established an animal model of 

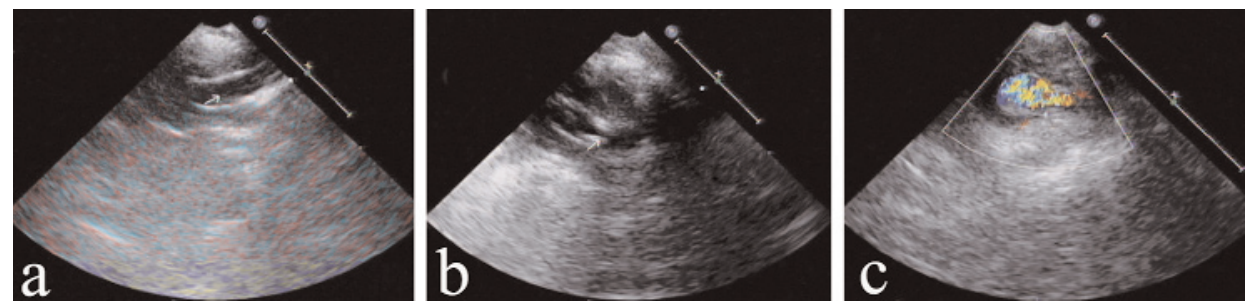

Fig. 1. Representative ultrasound images show aortic dilatation. a. The normal rat ascending aorta. b. Operated group, arrowheads show the site of banding. c. Operated group, the dilated ascending aorta

ascending aortic aneurysm in rats. Using ultrasonic and histological techniques, we studied the changes of morphology and hemodynamics in the dilated aorta. At 8 weeks after surgery, ultrasonography was performed on the rats. There was no dilation in the ascending aortas in the sham-operated group and the aortas were round and smooth. However, remarkable dilation was observed in the ascending aortas of the operated group, and the region of dilation was localized above the aortic banding ring. We found that after operation the aortas had a $44.26 \%$ increase in diameter as compared to sham group (sham group, $3.39 \pm 0.15 \mathrm{~mm}$; operated group, $2.35 \pm 0.05 \mathrm{~mm}, \mathrm{p}<0.05$ ) (Fig. 1, Fig. 2). With an arbitrary threshold of a $20 \%$ diameter increase considered aneurysmal dilation in this experimental model, 8 of 12 rats exhibited aneurysms in the operated group $(66.67 \%)$.

The histological changes of the dilated aorta were examined by light microscopy and scanning electron microscopy. HE staining showed that three layers, tunica intima, tunica media and tunica adventitia, were clearly observed in the normal ascending aortic wall (Fig. 3a). However, the aortic wall in the operated group was disorganized and infiltrated by many inflammatory cells (Fig. 3b). In parallel with aortic dilatation, major changes were accompanied in vascular elastin integrity as shown by Romeis staining. Compared with the sham-operated group (Fig. 3c), aortic elastin in the operated group exhibited characteristic flattening and fragmentation (Fig. 3d). Sometimes, the fragmented elastic laminae were infiltrated by inflammatory cells (Fig. 3e, f).

\section{Expression of CD45, MCP-1, ICAM-1 and VCAM-1 in the ascending aorta}

To evaluate vascular inflammation, immunostaining of $\mathrm{CD}^{+} 5^{+}$(pan-leukocyte marker) was conducted. The density of leukocytes was determined by counting the mean number of nuclei surrounded by positive immunostaining within $400 \times$ high power fields (HPF). In the normal arterial wall, $\mathrm{CD} 45^{+}$expression was only slightly detected in the intima of the vascular wall. In contrast, in aneurysmal aorta, $\mathrm{CD} 45^{+}$cells were present in all layers of the vascular wall, suggesting aneurysmal aortas had greater infiltration as compared to normal aortas (Fig. 4).

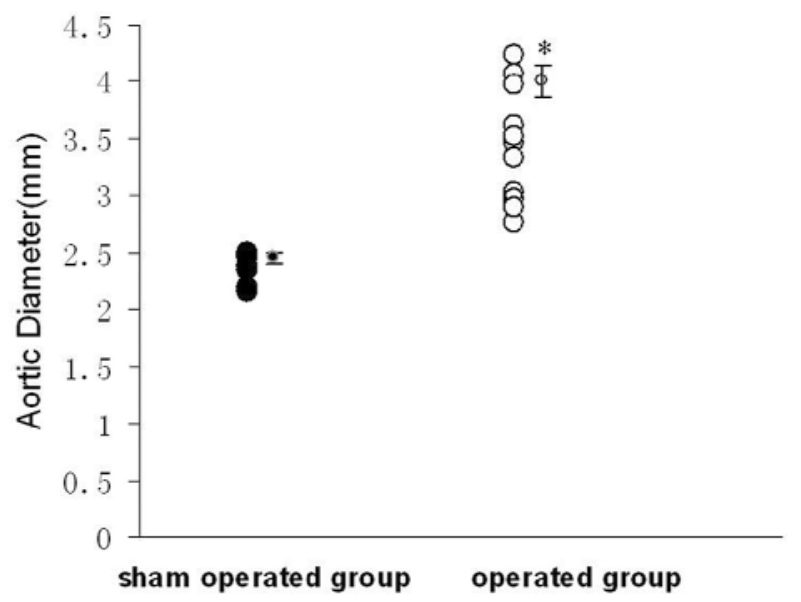

Fig. 2. Comparison of sham-operated and operated rat ascending aortic diameter. ${ }^{*} \mathrm{p}<0.05$ versus sham ascending aorta.

To determine the immunolocalization of MCP-1, ICAM-1 and VCAM-1 in the ascending aorta, immunohistochemical staining techniques were used. There was very little immunostaining of MCP-1, ICAM-1 and VCAM-1 in the normal ascending aorta (Fig. 5a1, b1, c1). In the operated group, the immunostaining of MCP-1 was markedly increased and localized in the intima of the ascending aorta (Fig. 5a2). Most VCAM-1 immunostaining was mainly localized in the endothelium of the neointimal thickening regions (Fig. 5b2). ICAM-1 was most intensively immunostained in the luminal endothelium, while the immunostaining was scattered in the media and adventitia of the ascending aorta (Fig. 5c2). The mean optical density of MCP-1, ICAM-1 and VCAM-1 was significantly increased in the operated group as compared to the sham group (Fig. 5d).

\section{Expression of MMP-2 and MMP-9}

In a variety of MMPs, MMP-2 and MMP-9 have been considered to be especially important in the pathogenesis of the aortic aneurysm [20,21]. Gelatin zymography is a sensitive technique that detects activated gelatin-degrading metalloproteinases. The activity and expression of MMP-2 and MMP-9 of operated group was significantly increased compared with the sham group (Fig. 6). These results indicate that MMP-2 and MMP-9 are involved in the development of aneurysm. 

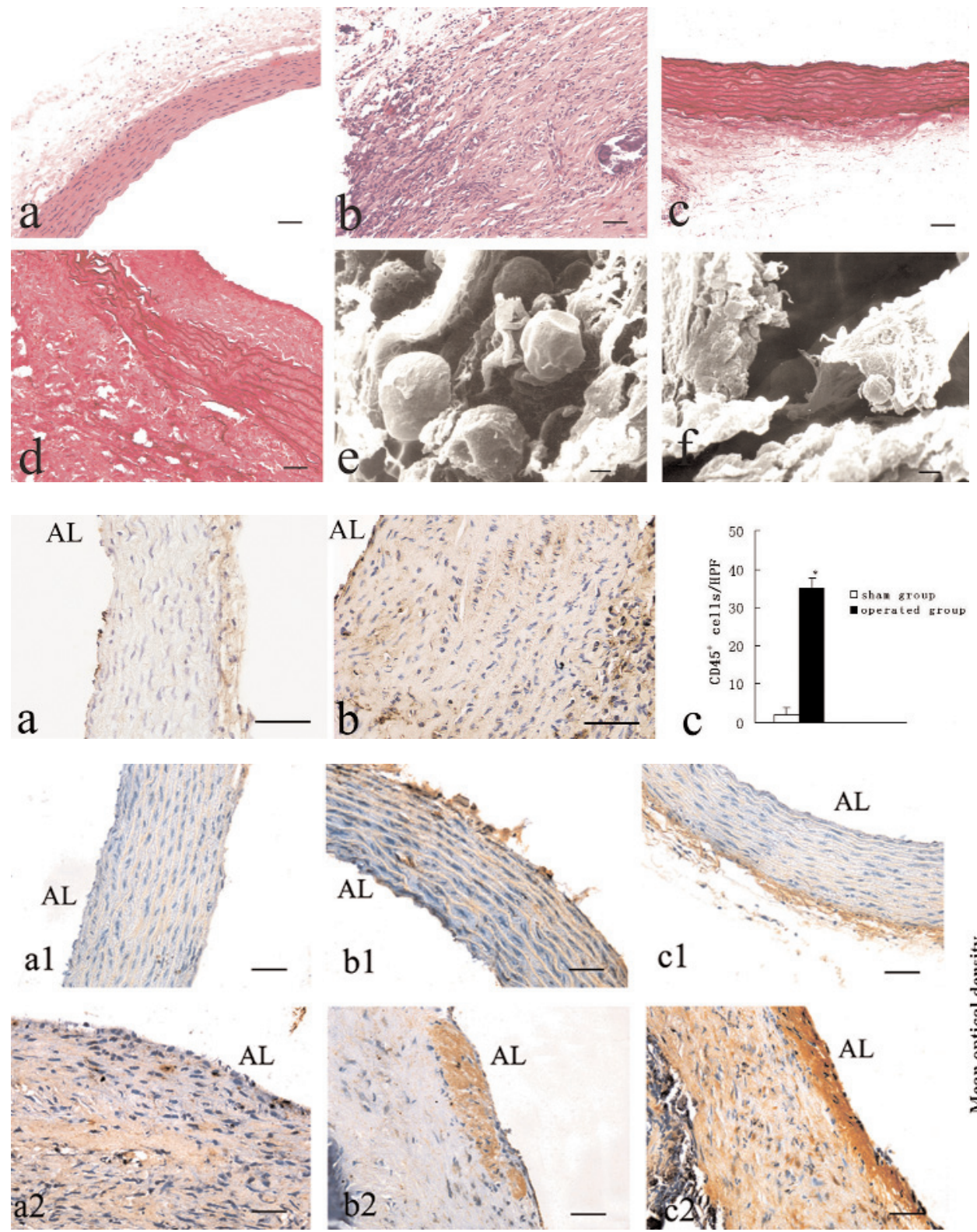

Fig. 3. Histology of rat aorta. a. HE staining shows the wall of the normal ascending aorta. b. HE staining shows the wall of the dilated ascending aorta. c. Romeis staining shows elastic lamellae in the wall of the normal ascending aorta. d. Romeis staining shows elastic lamella in the wall of the dilated ascending aorta. e. The inflammatory cells infiltrating the wall of the dilated ascending aorta. f. The inflammatory cells infiltrating the fragmented sites of the elastic lamella of the dilated ascending aorta. Scale bars: $50 \mu \mathrm{m}$ (a-d) and $1 \mu \mathrm{m}(\mathrm{e}, \mathrm{f}),(\mathrm{n}=5)$.

Fig. 4. Immunohistochemical staining of CD45 (a, b) and number of migrated leukocytes in vessel wall after 8 weeks of treatment (c). Brown deposits indicate positive staining. a. The wall of the normal ascending aorta. $b$. The wall of the dilated ascending aorta. AL: aortic lumen, Scale bars: $50 \mu \mathrm{m}$. $(\mathrm{n}=8)$

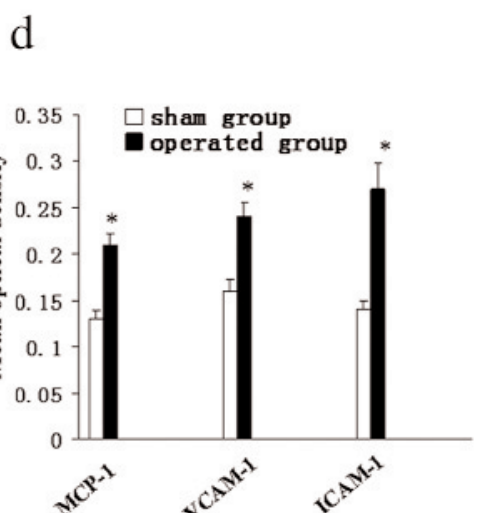

Fig. 5. Immunohistochemical staining of MCP-1, VCAM-1 and ICAM-1. Brown deposits indicate positive staining. a1, a2. Immunostaining of MCP-1. b1, b2. Immunostaining of VCAM-1. c1, c2. Immunostaining of ICAM-1. a1, b1, c1. The wall of the normal ascending aorta. a2, b2, c2. The wall of the dilated ascending aorta. AL: aortic lumen, Scale bars: $50 \mu \mathrm{m}$. d. Comparison of MCP-1, VCAM-1 and ICAM-1 mean optical density values of sham group and operated group. All values are mean \pm standard deviation (SD) ( $\mathrm{n}=6$ ). * $\mathrm{p}<0.05$ versus sham group.
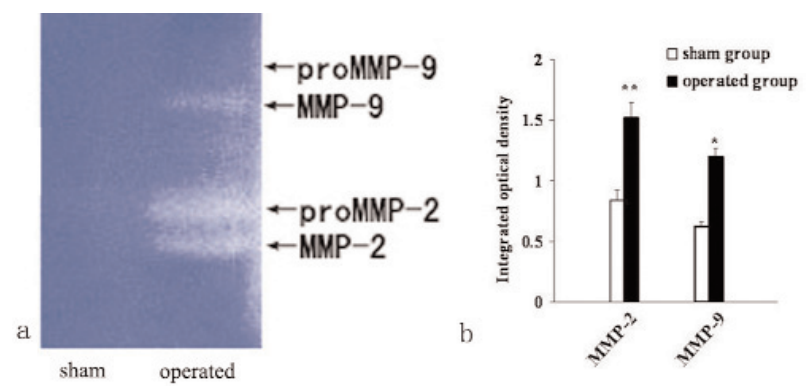

Fig. 6. Gelatin zymographic analysis of protein levels of MMP-2 and MMP-9 in the rat aorta (a). The densitometric analysis showing that the activity and expression of MMP-2 and MMP-9 of the operated group was significantly enhanced compared with the sham group (b). All values are mean \pm standard deviation (SD) $(\mathrm{n}=5) .{ }^{*} \mathrm{p}<0.05$ versus sham group, ${ }^{* *} \mathrm{p}<0.01$ versus sham group.
A strong relationship between vascular inflammation and the progression of abdominal aortic aneurysm has been reported from clinical findings and animal experiments $[22,23]$. While the immunological mechanisms underlying atherosclerosis and abdominal aortic aneurysm have been somewhat characterized, little is known about the role of inflammatory cells, cytokines, and cell adhesion molecules in the pathogenesis of ascending aortic aneurysm. In this study, we used experimental aneurysms in rat to study the immunolocalization of some cellular and extracellular molecules

\section{Discussion}


involved in the vascular remodeling of aneurysms. Banding of the ascending aorta of rats resulted in enlargement of the vessel. Specific arterial sites, such as branches, bifurcations, and curvatures, cause characteristic alterations in the flow of blood, including decreased shear stress and increased turbulence [24]. Cynomolgus monkeys that underwent mid-thoracic aortic coarctation led to produce post-stenotic dilatation [19]. Post-stenotic dilatation of the ascending aorta is seen in patients with aortic stenosis and/or aortic regurgitation [25]. This dilatation is usually progressive and may undergo aneurysmal degeneration with disruption of the artery wall [26]. The aorta has multiple cellular origins in the developing embryo. The origination of ascending aorta is different from the descending thoracic and abdominal aorta [27]. Thus, the present results extend the post-stenotic dilatation for the study of ascending aortic aneurysms.

In the present study, our results demonstrated that the formation of dilated ascending aorta in this model was associated with the expression of MCP-1, ICAM1, VCAM-1, and MMPs and infiltration of inflammatory cells. We found many inflammatory cells in aneurysm walls from histological evidence and immunohistochemical staining of CD45. CD $45^{+}$cells were present in all layers of the vascular wall. Recent studies revealed that inflammation contributed to ascending aortic aneurysm formation. Immunohistochemical studies in ascending aortic aneurysm demonstrated that the numbers of $\mathrm{T}$ lymphocytes and macrophages were increased compared with normal aortas $[4,5]$.

The expression of leukocyte adhesion molecules on endothelial cells, such as ICAM-1 and VCAM-1, plays an important role in the mediation of the location of monocytes at the intimal surface $[21,28-30]$. From our observations of ICAM-1 and VCAM-1 immunolocalization in ascending aortic tissues, very low or no expression of ICAM-1 and VCAM-1 was seen in normal ascending aortas, but in situ ICAM-1 and VCAM1 expression was significantly increased in aneurysmal dilatation tissues. The enhanced expressions of ICAM1 and VCAM-1 in aneurysmal dilatation tissues confirm the involvment of inflammation in aneurysm. The expression of VCAM-1 was up-regulated in the endothelium of rat cerebral aneurysms in the early stage of cerebral aneurysm formation, while inhibiting the upregulation of VCAM- 1 expression in aneurysmal walls, consequently led to reduction of macrophage infiltration into aneurysmal walls [31]. Elevated levels of VCAM-1 on the vessel endothelial surface may play an important role in ascending aortic aneurysm development by leukocytes recruitment. ICAM-1 interacts with leukocytes principally by binding to the surface membrane beta-2 integrin molecule, LFA-1 [32]. ICAM-1 expression was up-regulated in abdominal aortic aneurysm compared with normal aortic tissues [30]. In our results, endothelial expression of ICAM-1 may be involved in the recruitment of leucocytes to the lesion as suggested by its role in the entry of leukocytes into foci of inflammation.

In atherosclerosis, endothelial dysfunction induces monocyte recruitment by transcriptional regulation of various proinflammatory genes, such as ICAM-1 and VCAM-1 [28,29,33]. Although the role of endothelial cells in ascending aortic aneurysm formation has not yet been established, a similar mechanism may be involved in the initiation of ascending aortic aneurysm formation. We observed that MCP-1 was increased and localized in the intima of the ascending aorta in the operated group. MCP-1 is related to monocyte/ macrophage recruitment, which accumulates in the injured region in various vascular diseases, such as atherosclerosis $[34,35]$ and abdominal aortic aneurysm [36-38]. MCP-1-/- mice exhibited a significant decrease in cerebral aneurysm formation and macrophage accumulation as well as a decrease in the expression of MMP-2 and MMP-9 [39]. Our data suggest that MCP-1 may play important roles in ascending aortic aneurysm formation by recruiting monocyte/macrophage into aneurysmal wall.

In addition to dilatation, damage of arterial elastin is also a pathogenic aspect characteristic of the ascending aortic aneurysms. We noted that activation and expression of MMP-2 and MMP-9 in the arterial walls of early aneurysmal changes was detected at 8 weeks of aneurysm induction with the enhanced expression of MCP-1, ICAM-1 and VCAM-1. These findings suggest that an active vascular response is present in the early aneurysmal dilatation. MMPs are calciumand zinc-dependent endopeptidases, are able to degrade extracellular components and play a fundamental role in tissue remodeling [40]. Recently, we have demonstrated that MMP-2, MMP-3 and MMP-9 were activated in 3-5 months after ascending aortic aneurysm induction $[17,18]$. In the transwell experiment, the up-regulation of MMP-9 in T lymphoma cells upon contacting with endothelium required ICAM-1-mediated adhesion [41]. In atherogenesis, the expression and activity of MMP-2 and MMP-9 was up-regulated by VCAM-1, while MMP inhibitors blocked this act [42].

To further clarify the role of MCP-1, ICAM-1 and VCAM-1 in ascending aortic aneurysm formation, knock-out animal models will be used in the future studies. In summary, the data presented in this study provide further evidence to prove that inflammatory processes are involved in the pathogenesis of ascending aortic aneurysm.

Acknowledgements: The authors are grateful for generous assistance by Deshou Cao from the Institute of Pharmacology, Southern Illinois University School. 


\section{References}

[1] Bickerstaff LK, Pairolero PC, Hollier LH, Melton LJ, Van Peenen HJ, Cherry KJ et al., Thoracic aortic aneurysms: a population-based study. Surgery. 1982;92;1103-1108.

[2] Jones JA, Spinale FG, Ikonomidis JS. Transforming growth factor-beta signaling in thoracic aortic aneurysm development: a paradox in pathogenesis. J Vasc Res. 2009;46:119137.

[ 3] Tang PC, Yakimov AO, Teesdale MA, Coady MA, Dardik A, Elefteriades JA et al. Transmural inflammation by interferongamma-producing $\mathrm{T}$ cells correlates with outward vascular remodeling and intimal expansion of ascending thoracic aortic aneurysms. FASEB J. 2005;19:1528-1530.

[ 4] He R, Guo DC, Estrera AL, Safi HJ, Huynh TT, Yin Z et al. Characterization of the inflammatory and apoptotic cells in the aortas of patients with ascending thoracic aortic aneurysms and dissections. J Thorac Cardiovasc Surg. 2006; 131:671-678

[ 5] He R, Guo DC, Sun W, Papke CL, Duraisamy S, Estrera AL et al., Characterization of the inflammatory cells in ascending thoracic aortic aneurysms in patients with Marfan syndrome, familial thoracic aortic aneurysms, and sporadic aneurysms. $J$ Thorac Cardiovasc Surg. 2008;136:922-929.

[6] Gong Y, Hart E, Shchurin A, Hoover-Plow J. Inflammatory macrophage migration requires MMP-9 activation by plasminogen in mice. J Clin Invest. 2008;118:3012-3024.

[7] Brophy CM, Reilly JM, Smith GJ, Tilson MD. The role of inflammation in nonspecific abdominal aortic aneurysm disease. Ann Vasc Surg. 1991;5:229-233.

[ 8] Milioti N, Bermudez-Fajardo A, Penichet ML, Oviedo-Orta E. Antigen-induced immunomodulation in the pathogenesis of atherosclerosis. Clin Dev Immunol. 2008;2008:723539.

[9] Collier IE, Smith J, Kronberger A, Bauer EA, Wilhelm SM, Eisen AZ et al. The structure of the human skin fibroblast collagenase gene. J Biol Chem. 1988;263:10711-10713.

[10] Xu QB, Oberhuber G, Gruschwitz M, Wick G. Immunology of atherosclerosis: cellular composition and major histocompatibility complex class II antigen expression in aortic intima, fatty streaks, and atherosclerotic plaques in young and aged human specimens. Clin Immunol Immunopathol. 1990;56: 344-359.

[11] Katsuda S, Boyd HC, Fligner C, Ross R, Gown AM. Human atherosclerosis. III. Immunocytochemical analysis of the cell composition of lesions of young adults. Am J Pathol. 1992; 140:907-914

[12] Bobryshev YV, Lord RS, S-100 positive cells in human arterial intima and in atherosclerotic lesions. Cardiovasc Res. 1995;29:689-696.

[13] Millonig G, Malcom GT, Wick G. Early inflammatoryimmunological lesions in juvenile atherosclerosis from the Pathobiological Determinants of Atherosclerosis in Youth (PDAY)-study. Atherosclerosis. 2002;160:441-448.

[14] Ocana E, Perez-Requena J, Bohorquez JC, Brieva JA, Rodriguez C. Chemokine receptor expression on infiltrating lymphocytes from abdominal aortic aneurysms: role of CXCR4-CXCL12 in lymphoid recruitment. Atherosclerosis. 2008;200:264-270

[15] Kaufmann BA, Sanders JM, Davis C, Xie A, Aldred P, Sarembock IJ et al. Molecular imaging of inflammation in atherosclerosis with targeted ultrasound detection of vascular cell adhesion molecule-1. Circulation. 2007;116:276-284.

[16] Rollins BJ. Chemokines. Blood. 1997;90:909-928.

[17] Fan J, Bai SL, Zhu XB. Expression of matrix metalloproteinase-3, matrix metalloproteinase-9 in the ascending aortic aneurysm. Chin J Histochem Cytochem. 2005a;5:536-539.

[18] Fan J, Bai SL, Cao DS., Wang W, Zhu XB. Morphological study on ascending aortic aneurysms induced by ascending aorta banding. Acta Anatomic Sinica. 2005b;36:442-446.

[19] Zarins CK, Runyon-Hass A, Zatina MA, Lu CT, Glagov S. Increased collagenase activity in early aneurysmal dilatation. J Vasc Surg. 1986;3:238-248.

[20] Longo GM, Xiong W, Greiner TC, Zhao Y, Fiotti N, Baxter BT. Matrix metalloproteinases 2 and 9 work in concert to produce aortic aneurysms. J Clin Invest. 2002;110:625-632.

[21] Sadek M, Hynecek RL, Goldenberg S, Kent KC, Marin ML, Faries PL. Gene expression analysis of a porcine native abdominal aortic aneurysm model. Surgery. 2008;144:252258.

[22] Freestone T, Turner RJ, Coady A, Higman DJ, Greenhalgh RM, Powell JT. Inflammation and matrix metalloproteinases in the enlarging abdominal aortic aneurysm. Arterioscler Thromb Vasc Biol. 1995;15:1145-1151.

[23] Vainas T, Lubbers T, Stassen FR, Herngreen SB, van DieijenVisser MP, Bruggeman CA et al. Serum C-reactive protein level is associated with abdominal aortic aneurysm size and may be produced by aneurysmal tissue. Circulation. 2003;107:1103-1105.

[24] Weissberg PL, Bennett MR. Atherosclerosis--an inflammatory disease. $N$ Engl J Med. 1999;340:1928-1929.

[25] Keane MG, Wiegers SE, Plappert T, Pochettino A, Bavaria JE, Sutton MG. Bicuspid aortic valves are associated with aortic dilatation out of proportion to coexistent valvular lesions. Circulation. 2000;102:III35-39.

[26] Wilton E, Bland M, Thompson M, Jahangiri M. Matrix metalloproteinase expression in the ascending aorta and aortic valve. Interact Cardiovasc Thorac Surg. 2008; 7:37-40.

[27] Ikonomidis JS, Gibson WC, Gardner J, Sweterlitsch S, Thompson RP, Mukherjee R et al. A murine model of thoracic aortic aneurysms. J Surg Res. 2003;115:157-163.

[28] Constans J, Conri C. Circulating markers of endothelial function in cardiovascular disease. Clin Chim Acta. 2006;368:33-47.

[29] Rao RM, Yang L, Garcia-Cardena G, Luscinskas FW. Endothelial-dependent mechanisms of leukocyte recruitment to the vascular wall. Circ Res. 2007;101:234-247.

[30] Szekanecz, Z., Shah, M.R., Pearce, W.H., Koch, A.E.: Increased intercellular adhesion molecule-1 (ICAM-1) expression and soluble ICAM-1 (sICAM-1) production by cytokine-activated human aortic endothelial cells: a possible role for ICAM-1 and sICAM-1 in atherosclerotic aortic aneurysms. Clin Exp Immunol. 1994; 98:337-343.

[31] Aoki T, Kataoka H, Ishibashi R, Nozaki K, Hashimoto N. Simvastatin suppresses the progression of experimentally induced cerebral aneurysms in rats. Stroke. 2008; 39:12761285.

[32] Poston RN, Haskard DO, Coucher JR, Gall NP, JohnsonTidey RR. Expression of intercellular adhesion molecule-1 in atherosclerotic plaques. Am J Pathol. 1992;140:665-673.

[33] Fisker Hag AM, Pedersen SF, Kjaer A. Gene expression of LOX-1, VCAM-1, and ICAM-1 in pre-atherosclerotic mice. Biochem Biophys Res Commun. 2008;377: 689-693.

[34] Egashira K. Molecular mechanisms mediating inflammation in vascular disease: special reference to monocyte chemoattractant protein-1. Hypertension. 2003;41:834-1841.

[35] Linton MF, Fazio S. Macrophages, inflammation, and atherosclerosis. Int J Obes Relat Metab Disord. 2003;27:Suppl 3:S35-40.

[36] Ishibashi M, Egashira K, Zhao Q, Hiasa K, Ohtani K, Ihara Y et al. Bone marrow-derived monocyte chemoattractant protein-1 receptor CCR2 is critical in angiotensin II-induced acceleration of atherosclerosis and aneurysm formation in hypercholesterolemic mice. Arterioscler Thromb Vasc Biol. 2004;24:174-178.

[37] Golledge J, Muller J, Daugherty A, Norman P. Abdominal aortic aneurysm: pathogenesis and implications for management. Arterioscler Thromb Vasc Biol. 2006;26:2605-2613. 
[38] Middleton RK, Bown MJ, Lloyd GM, Jones JL, London NJ, Sayers RD. Characterisation of Interleukin- 8 and monocyte chemoattractant protein-1 expression within the abdominal aortic aneurysm and their association with mural inflammation. Eur J Vasc Endovasc Surg. 2009;37:46-55.

[39] Aoki T, Kataoka H, Ishibashi R, Nozaki K, Egashira K, Hashimoto N. Impact of monocyte chemoattractant protein-1 deficiency on cerebral aneurysm formation. Stroke. 2009;40: 942-951.

[40] Chow AK, Cena J, Schulz R. Acute actions and novel targets of matrix metalloproteinases in the heart and vasculature. $\mathrm{Br}$ J Pharmacol. 2007;152:189-205.
[41] Aoudjit F, Potworowski EF, St-Pierre Y. Bi-directional induction of matrix metalloproteinase-9 and tissue inhibitor of matrix metalloproteinase-1 during $\mathrm{T}$ lymphoma/endothelial cell contact: implication of ICAM-1. J Immunol. 1998;160: 2967-2973.

[42] Fang ZY, Lin R, Yuan BX, Liu Y, Zhang H. Tanshinone IIA inhibits atherosclerotic plaque formation by down-regulating MMP-2 and MMP-9 expression in rabbits fed a high-fat diet. Life Sci. 2007;81:1339-1345.

Submitted: 29 January, 2010 Accepted after reviews: 1 June, 2010 